PAPER

\title{
Retransmission Diversity with Channel Selectivity for High Reliable and Low Latency Industrial Wireless Control System
}

\author{
Astri Maria Kurniawati ${ }^{1}$, Nana Sutisna ${ }^{2}$, Leonardo Lanante $\mathrm{Jr}^{2}$, Yuhei Nagao ${ }^{2}$, Masayuki Kurosaki ${ }^{2}$ \\ and Hiroshi Ochi ${ }^{2}$
}

\author{
${ }^{1}$ Graduate School of Computer Science and Electronics, Kyushu Institute of Technology \\ ${ }^{2}$ Department of Computer Science and Electronics, Kyushu Institute of Technology \\ 680-4 Kawazu, Iizuka, Fukuoka 820-8502, Japan \\ E-mail: astri@dsp.cse.kyutech.ac.jp, ochi@cse.kyutech.ac.jp
}

\begin{abstract}
The emerging applications in the 5th generation (5G) network, such as industrial control system and Machine-toMachine (M2M) communication require high reliability and low latency communication at the same time. The retransmission scheme is one of the practical approaches to increase system reliability when other diversity techniques are avoided due to hardware complexity or significant transmission delay. In order to address such problems, in this paper, we propose a fast yet reliable retransmission scheme. The proposed retransmission scheme leverages the frequency diversity through simple channel selectivity. The channel selectivity is carried out by employing low complexity subcarrier allocation for selecting other channels in Orthogonal Frequency Division Multiplexing (OFDM) transmission. The evaluation results show that the proposed retransmission method outperforms the conventional scheme. The proposed method can reduce the number of retransmission by up to $30 \%$, corresponding to a success rate of 0.9999 . In addition, it can be employed in an industrial Wireless Local Area Network (iWLAN) system with a large number of users. The achievable control duration is around 52-63 $\mu \mathrm{s}$, an improvement of approximately $36 \%$ compared to the conventional system.
\end{abstract}

Keywords: fast retransmission, channel selectivity, frequency diversity, industrial wireless system

\section{Introduction}

In the new vision of Industry 4.0 era and 5G network, wireless system has been considered as a potential solution for industrial communication due to its primary advantages in terms of flexibility for controlling mobile clients and the capability for reducing physical connectivity in a factory environment [1]. In the industrial control application, the main Quality of Service (QoS) requirements are ultra reliable and low latency communication (URLLC), deterministic communication, and support for high dense networks rather than high throughput metrics [2].

The deployment of wireless networks in industrial communication systems have been considered for Factory Automation (FA) application. These include Bluetooth, ZigBee, and WirelessHART [3]-[6]. However, those existing technologies suffer from the strict requirements of FA communication system, particularly for achieving high reliability with fast control duration. For example, while wirelessHART can achieve high reliability in terms of Packet Error Rate (PER) of $10^{-8}$ to $10^{-9}$, it suffers from high latency. Therefore, achieving both high reliability and low latency requirements is still a challenging task in industrial wireless environment, specifically due to many obstacles and interference [7].

In order to increase system reliability, there are several well known and established practical approaches. These include employing spatial diversity techniques for example Multiple Input Multiple Output (MIMO) and advanced error correction mechanisms such as Low Density Parity Check (LDPC) and Turbo Coding. In other cases, a parallel redundant system could also be deployed to increase system reliability [8]. Indeed, these techniques result in higher implementation complexity and increasing power consumption which is not favorable for deployment in an industrial environment.

Retransmission that leverages temporal diversity is a common practical approach to increase system reliability. A conventional Automatic Repeat Request (ARQ) scheme has been employed as a retransmission scheme in the previous iWLAN system [9]. This system is also known as FAWLAN system. However, this communication protocol is frequency oblivious, i.e. the frequency diversity has not been exploited yet. Hence, when the system perfor- 
mance suffers from a persistently bad channel condition, the retransmission cannot recover from communication error [10]. In particular, the retransmission only gives reliability improvement if the channel has changed into a better state. As a result, the system reliability improvement is limited in a typical industrial wireless network where the channel characteristics are relatively steady [11]. As a result, the system failure will occur frequently.

In order to solve the persistent channel problem, another option is to select other channels by performing channel scanning and re-tuning center frequency $(\mathrm{CF})$ of Radio Frequency (RF) circuit. Unfortunately, performing frequent $\mathrm{CF}$ switching requires significant setup time, which is around an order of several milliseconds. This approach will introduce extra overhead and degrade system performance in terms of fast control duration. Therefore, it is not suitable for time critical communication needed for industrial control.

Another alternative solution is performing channel selection by utilizing feedback information such as Channel State Information (CSI) from the receiver [12]. However, acquiring such information requires channel sounding, which involves intensive data exchange between Access Point (AP) and Station (STA). This results in a higher system complexity and also gives significant communication overhead.

Considering the limitations on the conventional retransmission scheme, we propose exploiting the frequency diversity to achieve high reliability performance while maintaining overall system latency. The main contributions of this work are thus summarized as follows:

a) High reliability system: Improvement on system reliability is achieved by exploiting the opportunity of frequency diversity in a wider channel bandwidth through channel selectivity.

b) Fast retransmission scheme: The retransmission procedure is done by performing subcarrier allocation/de-allocation of Inverse Fast Fourier Transform (IFFT)/Fast Fourier Transform (FFT) in digital baseband domain. Thus we can avoid RF recalibration and sounding procedure to obtain CSI.

c) Low complexity implementation: The proposed method only needs to implement additional circuits for handling subcarrier allocation and does not require intensive feedback information from the upper layers.

d) Furthermore, in order to evaluate the applicability of the proposed scheme in a real system, we also apply the proposed retransmission scheme into the FAWLAN system. The performance evaluations, including system-level performance are also presented.

The rest of the paper is organized as follows. Firstly, we review the FAWLAN communication system as well as the proposed protocol and transmission schemes in Section 2. Then, Section 3 explains the system model and elaborates the requirements of channel condition for selectivity scheme. In Section 4, the proposal of fast and low complexity retransmission is presented. Section 5 describes models of performance metrics used in the paper. The results, in terms of reliability and latency are discussed in Section 6. Finally, concluding remarks are given in Section 7.

\section{Overview of Industrial Wireless System}

\subsection{General communication protocol of FAWLAN system}

In order to obtain a deterministic communication, we consider a synchronous multiuser transmission protocol, where one AP serves multiple STAs (e.g. industrial robots), as shown in Fig. 1.

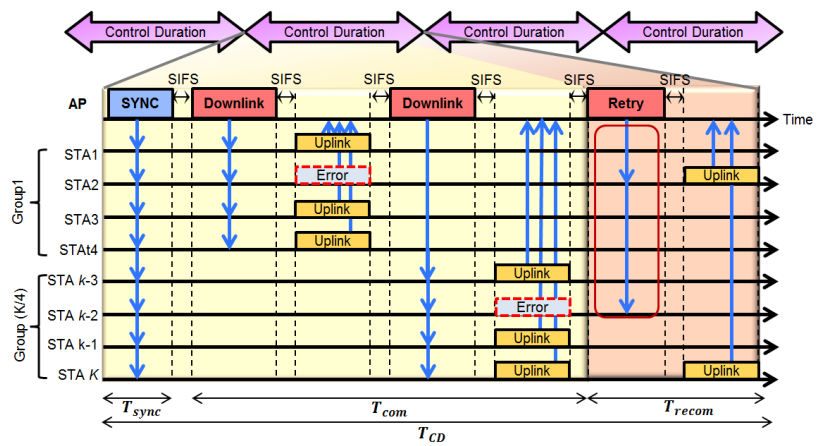

Fig. 1 FAWLAN system transmission protocol

The transmission operation mainly consists of three stages: initialization, communication, and retransmission. In the initialization stage, the AP initiates a new control duration $\left(T_{C D}\right)$ by broadcasting a synchronization (SYNC) frame to all stations (STAs), using certain transmission bandwidth which can vary depending on the number of associated STAs in one control duration. The SYNC frame may include the Precision Time Protocol (PTP) information for synchronization purposes. As shown in Fig. 1, the SYNC frame occupies the channel for a period of $T_{\text {sync }}$.

After all STAs have been synchronized, the AP performs communication with all $K$ STAs for a duration of $T_{\text {com }}$. This communication phase incorporates two operations: Downlink (DL) transmission and Uplink (UL) transmission. The DL and UL transmissions are performed until the AP finishes delivering data to all $K$ STAs. When the STA either fails receiving the DL packet from the AP or transmitting the UL packet to the AP, an error is said to have occured.

If UL transmission error occurred during normal communication stage (as illustrated by STA 2 and STA $k-2$ in Fig. 1), the STA will retransmit the unsuccessful packet to the AP. Principally, the operation in this stage is the same as the normal communication process. This recommunication duration is denoted by $T_{\text {recom }}$. The retransmission process could be repeated until the AP successfully receives packet from all $K$ STAs or has reached the $T_{C D}$ duration. 
When the $T_{C D}$ elapses and there is no STA error in this stage, a new $T_{C D}$ could be performed. The number of times required to perform the retransmission stage is defined as $\Gamma$. If there is still any STA error after $\Gamma$ transmissions (i.e. after $T_{C D}$ elapses), the system will be considered to be faulty and should be terminated immediately.

\subsection{FAWLAN transmission scheme}

In order to support low overhead communication, FAWLAN system employs multiple access transmission schemes, which are Multi User - Packet Division Multiple Access (MU-PDMA) for DL communication and Frequency Division Multiple Access (FDMA) scheme for UL communication, similar with [9]. For clarity, Fig. 2 and Fig. 3 illustrate the transmission schemes on FAWLAN system for DL (80 MHz bandwidth) and UL (4 STAs) respectively.

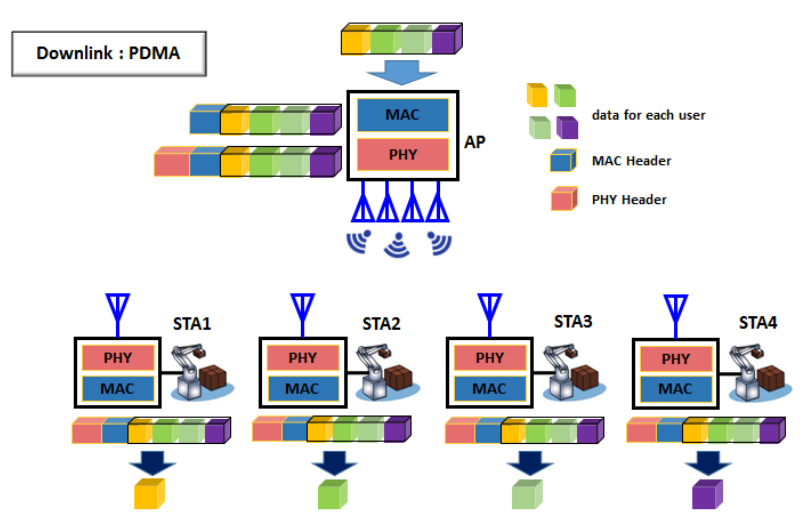

Fig. 2 PDMA transmission scheme

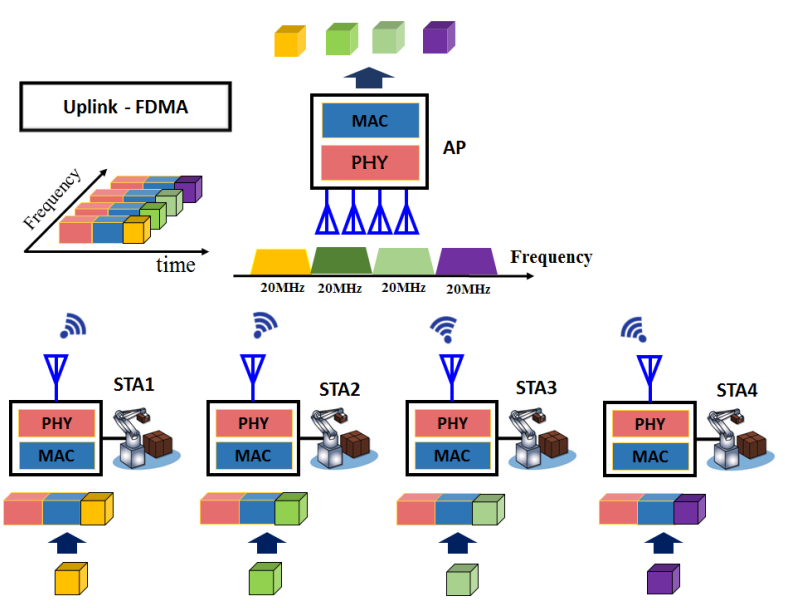

Fig. 3 FDMA transmission scheme

In DL transmission, the packet data of Medium Access layer (MAC) referred to as MAC Service Data Unit (MSDU) data of users, are merged into one transmission frame (MSDU1 - MSDU4). The MAC header and Physical layer (PHY) header fields that contain transmission con- figuration are also appended into this DL frame. Subsequently, this MU-PDMA frame is transmitted to all users on the same channel bandwidth of $80 \mathrm{MHz}$. Additionally, this MU-PDMA frame could also be transmitted to all users through multiple antenna streams to obtain the diversity gain. In the receiver of each user, the MSDU is extracted according to the user parameters specified in the header field of its received frame.

In the UL transmission, on the other hand, multiple users (STAs) simultaneously transmit data using different channels. In this scheme, each STA occupies $20 \mathrm{MHz}$ channel bandwidth and the system allows 4 users to use the total channel bandwidth of $80 \mathrm{MHz}$. The allocated channel number for each STA is obtained from UL CHANNEL field of the received MU-PDMA frame. It should be noted that the conventional FAWLAN transmission uses a fixed channel for configuring RF front end and performs normal UL transmission and retransmission, unless the AP changes the UL CHANNEL information.

In order to enable retransmission diversity with channel selectivity scheme, the transmission protocol of FAWLAN is slightly modified. The UL CHANNEL information that is sent by the AP during DL transmission is expanded to add the information of initial subchannel for subcarrier allocation. When retransmission stage is required, STA and AP will automatically (controlled by digital hardware circuit) switch to the next channel to gain the opportunity of frequency diversity. Therefore, this scheme reduces the overhead in communication duration and saves processing time for RF circuit setup.

\section{System Model and Channel Characteristic Requirements}

\subsection{System model}

Without loss of generality, we consider a simplified FDMA UL system, where $K$ users transmit simultaneously to one AP in one communication cycle. Each user takes payload data $b_{i}$ and this lower-rate stream is scrambled, encoded by Forward Error Correction (FEC), and interleaved independently. The resulting bit-streams are then mapped to constellation points using Quadrature Amplitude Modulation (QAM) modulation, depending on the employed Modulation Coding Scheme (MCS). The resulting constellation points of data stream are considered as subcarrier $X_{i}$ and subsequently will be converted into parallel data as sets of OFDM symbols. Each OFDM symbol is then modulated using IFFT, creating a transmitted signal $x(n)$ in the time-domain, which can be expressed by

$$
x(n)=\sum_{i=0}^{N-1} X(i) e^{j(2 \pi i n / N)}
$$

where $N$ denotes FFT point, $n$ denotes index of sample data in time-domain, and $i$ represents subcarrier index of frequency-domain data. 
Before transmission over wireless channel, a cyclic prefix is appended by an amount of $N_{g}$ samples, resulting in the final transmitted signal $s(n)$ :

$$
s(n)= \begin{cases}x(n+N) & \text { for }-N_{g} \leq n<0 \\ x(n) & \text { for } 0 \leq n<N\end{cases}
$$

where $N_{g}$ denotes length of guard interval (in samples).

In the proposed work, we mainly focus on subcarrier allocation before OFDM transmission. Let the total subcarrier in the entire channel bandwidth be equal to the number of employed FFT points which is $N$, each user is assigned to occupy $N_{s c}$ subcarrier for FDMA transmission. Hence, the total available subchannel for UL transmission can be expressed as

$$
N_{s c h}=\frac{N}{N_{s c}}
$$

\subsection{Channel requirements for channel selectivity}

In this section, we assess and further explain the channel characteristics in industrial wireless environment in order to validate whether the proposed retransmission scheme is viable to be employed. We use the WLAN channel of TGn channel model [13] for this evaluation. Some practical parameters, such as RF frequency, moving speed, control duration time are taken into consideration to provide realistic assumptions.

(1) Slow Fading vs. Fast Fading. A channel can be categorized as slow or fast fading to quantify the rate at which the channel changes. The coherence time $T_{c}$ is considered as a parameter that determines whether a channel is considered slow or fast. It represents the time duration where the channel impulse response is considered not varying. In other words, the channel response value will not be correlated from the previous value. In addition, a slow or fast fading channel also depends on the communication delay which is application dependent.

A channel is considered as slow fading when the coherence time is large relative to the delay requirements of the application. This implies that during a specified transmission duration, the channel will roughly be constant. On the other hand, a fast fading channel occurs when the coherence time is small compared to the delay of the application.

In [14], $T_{c}$ could be approximated by:

$$
T_{c} \approx \frac{0.423}{D_{s}}
$$

where the $f_{d}$ represents Doppler spread that is determined by moving speed object and RF wavelength (e.g. RF carrier frequency). Moreover, the Doppler spread, $D_{s}$ could be obtained by using Eq. (4):

$$
D_{s}=\frac{v_{0}}{\lambda}
$$

where $v_{0}$ denotes the moving speed of robots, while $\lambda$ denotes the wavelength of the RF signal.
For a typical indoor scenario, the moving speed of factory automation robots (for example flexible robot arm) is around $0.4 \mathrm{~m} / \mathrm{s}$ [15] and the employed RF carrier frequency is $5.25 \mathrm{GHz}$. Therefore, the Doppler spread corresponds to $7.005 \mathrm{~Hz}$. This results in the coherence time of around $0.143 \mathrm{~s}$.

Considering FAWLAN communication protocol as the application target, which has a communication duration $\left(T_{\text {com }}\right)$ of $184 \mu \mathrm{s}$ and typical control duration period of 5 ms (including the retransmission time), it is clear that this communication delay is much lower than the coherence time. Hence, the channel can be considered to be in slow fading in this case. This implies that when the channel response is in deep fading, it is unlikely the channel will change immediately (time invariant) in the next retransmission slot. As a result, the conventional retransmission that only exploits temporal diversity will have poor error performance. This problem motivates our work to employ retransmission with channel selectivity that leveraging frequency diversity.

(2) Frequency Selective Fading. In order to obtain frequency diversity, the frequency selective fading should exist in the entire transmission bandwidth. The channel can be considered as frequency selective if the signal bandwidth is much larger than the coherence bandwidth. The coherence bandwidth reflects the range of frequencies over which the channel can be considered flat. In this case, a signal is likely to experience comparable or correlated amplitude fading. The coherence bandwidth, $B_{c}$ can be obtained from the delay spread characteristics (RMS value). The value is approximately equal to the reciprocal of delay spread, $T_{d s}[14]$ :

$$
B_{c} \approx \frac{1}{5 T_{d s}}
$$

As described in WLAN channel model [13], the delay spread will vary depending on the type of environment. In small area environments (channel models A, B and C) the delay spread varies from 0 to $30 \mathrm{~ns}$. On the other hand, in larger environments (channel model D, E, and F), the delay spread varies from 50 to $150 \mathrm{~ns}$. According to this delay spread range, the corresponding coherence bandwidth $\left(B_{c}\right)$ varies from $1.33 \mathrm{MHz}-4 \mathrm{MHz}$ as calculated by Eq. 5. This result shows that the coherence bandwidth range is smaller than the employed WLAN transmission bandwidth $(80 \mathrm{MHz})$ and thus confirms that the channels can be considered as frequency selective fading. Hence, the frequency diversity exists in the entire channel bandwidth, even for the smallest transmission bandwidth of WLAN system which is $20 \mathrm{MHz}$ bandwidth. This implies that the different frequency components of the signal experience uncorrelated fading.

Even with a frequency selective fading channel, it is highly unlikely that all parts of the signal will be simultaneously affected by a deep fade. Hence, we can expect that other channels separated by $20 \mathrm{MHz}$ has a better channel response allowing us to use the adjacent channel for re- 
transmission. According to these assessments, the channel selectivity scheme is potentially applicable to improve reliability performance of an industrial WLAN system.

\section{Proposed Retransmission with Channel Selectivity}

In principle, the proposed retransmission scheme could be employed for both DL and UL transmission. However, it is more relevant in the uplink where the transmitter node might not be interested in data decoding of other nodes [16]. In addition, in an industrial network environment, the transmission error can occur not only in the DL transmission, but also in the UL transmission [17]. Hence, the reliability of UL transmission is also a critical factor for achieving high system reliability. In this work, we mainly focus on UL transmission.

The transmission procedure and assumption used in the proposed transmission scheme can be summarized as follows:

a) The AP performs centralized access protocol in order to eliminate collision among STAs during UL transmission.

b) The AP configures the STAs to different subchannels by allocating corresponding data into specific subcarrier resources for the initial UL transmission.

c) The initial UL transmission channel is sent to each user within the DL PDMA frame.

d) The DL PDMA packet is sent using a robust modulation scheme to allow STA to decode subchannel fields for initial UL transmission.

e) The AP performs decoding of the initial UL transmissions from the STAs in their dedicated subcarrier and stores the successfully decoded signals.

f) For decoding retransmission UL packet, the AP attempts decoding the data on the next subchannel based on the predefined subchannel selection method.

\subsection{Channel selection methods}

Since this work is intended for industrial control system (e.g. FAWLAN system), where low latency and high reliability are primary requirements, we consider the retransmission method that offers low latency communication and low overhead (minimize unnecessary data transmission). Specifically, we avoid retransmission that requires feedback information (e.g. CSI or intensive control data exchange) and involves high-complexity processing.

In CSI based retransmission, such as in conventional WLAN system, the optimum channel selection is carried out after a sounding procedure that acquires channel state information. This scheme takes considerable time, around ten milliseconds order. Hence, this procedure results in a longer delay in transmission and should be avoided for industrial wireless systems.
To address this issue, such simple 'blind retransmissions' are considered in this paper. The channel selectivity is considered for retransmission procedure and it has been confirmed that this approach is applicable for WLAN channel characteristics, as previously described in section 3.2. Additionally, we also leverage the OFDM-based transmission, where we can manipulate subcarrier allocation of data before OFDM transmission for selecting subchannels. This results in quite a simple method (low complexity implementation) and offers faster processing time, as compared to other channel selection.

With these requirements, we considered retransmission with channel selectivity because of the following reasons:

(i) The proposed schemes can employ subcarrier allocation to select subchannels for retransmission. This allows low complexity implementation in designing PHY transceiver system and offers low processing latency. The necessary modifications on the transceiver design will be explained in section 4.2.

(ii) The retransmission could be performed directly without waiting for any feedback information. Therefore, communication overhead is significantly reduced and finally low latency communication is potentially achieved.

The two proposed schemes as illustrated in Fig. 4 include adjacent subchannel selection and random subchannel selection:

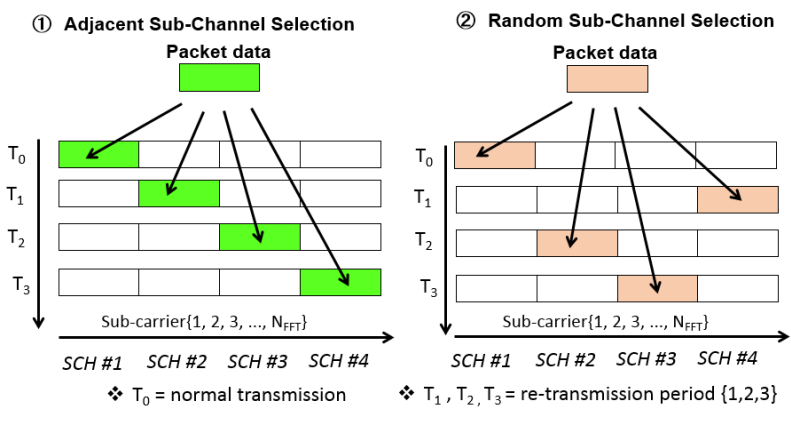

Fig. 4 Channel selectivity schemes for retransmission

1) Adjacent subchannel selection: In this scheme, channel selection is performed by immediately selecting the next adjacent subchannel from the previous transmission. Initially, the packet data for STA $k$ is assigned in the $k$-th subchannel based on initial UL subchannel configuration. For instance, STA1 occupies subchannel 1 and STA2-STA4 occupy subchannels 2-4, respectively. The data subcarrier is allocated to the corresponding subchannel index of the IFFT block and further goes to other transmitter blocks.

When the transmitted packet fails, the retransmission scheduler will automatically select the next adjacent subchannel. The index of subchannel, 
$\mathrm{SCH}_{\text {sel }}$, could be calculated using a simple approach, as expressed by Eq. (6).

$$
S C H_{a d j}(\tau)=S C H_{s e l}(\tau)=\bmod \left(\tau, N_{s c h}\right)+1
$$

where $\mathrm{SCH}_{\text {sel }}$ represents the current subchannel index for retransmission, $\tau$ denotes retransmission number, and $\bmod (a, b)$ is modulo operator that finds the remainder of division of $a$ by $b$.

2) Random subchannel selection: In this scheme, subchannel for next retransmission is selected as a random number from 1 up to $N_{s c h}$. The index of subchannel $S \mathrm{CH}_{\text {rand }}(t)$ is expressed in Eq. (7).

$$
S C H_{\text {rand }}(\tau)=S C H_{\text {sel }}(\tau)=R N D
$$

where $R N D$ is a random integer value and $R N D \in$ $\left\{1,2, \ldots, N_{\text {sch }}\right\}$.

We consider two types of random selections, which are random 1 and random 2 . In random 1 , the selected subchannel is carried out as true random and allows to reuse subchannel number. This case restricts the advantage of frequency diversity. On the other hand, in random 2 we avoid using subchannel that already employed in previous retransmission. Hence, all available subchannels will be utilized. In other words, the random 2 is similar with the adjacent channel method but using a different sequence index of subchannel.

\subsection{Transceiver design}

According to the proposed channel selection, the modification of the transceiver system is mainly on the subcarrier allocation part. In adjacent subchannel selection, the subcarrier index for retransmission is obtained by incrementing $N_{\text {sch }}$ from the previous allocated subcarrier. On the other hand, in random selection the value of $\tau$ will be picked randomly from $R N D \in\left\{1,2, \ldots, N_{s c h}\right\}$ instead of sequential values. Additionally, these two schemes can use a simple configurable address generator for allocating each subcarrier data to the corresponding FFT index.

In order to realize this retransmission scheme into an existing WLAN based transceiver system [18], the simple additional circuit and control unit are included to carry out subcarrier allocation by performing address generation for IFFT index, while the remaining blocks of the transmitter are unchanged.

The address generation is calculated based on the provided subchannel index $\mathrm{SCH}_{\text {sel }}$ from the control circuits. In particular, the control circuit handles the status signal from the upper layer that provides status of transmission, i.e. whether the data transmission is successful or failed. When the error transmission flag is received, the control signal will initiate retransmission process and ask the allocator to read data from the transmission packet buffer and assign the subcarrier data into another subchannel of IFFT index.
The overall modified transmitter structure is shown in Fig. 5. The transmitter data path modifications mainly include: (1) the subcarrier allocator block through implementation address generator, (2) Extension of FFT block to support up-to 256 point, (3) Control circuit to interpret status from upper layer and direct the retransmission process.

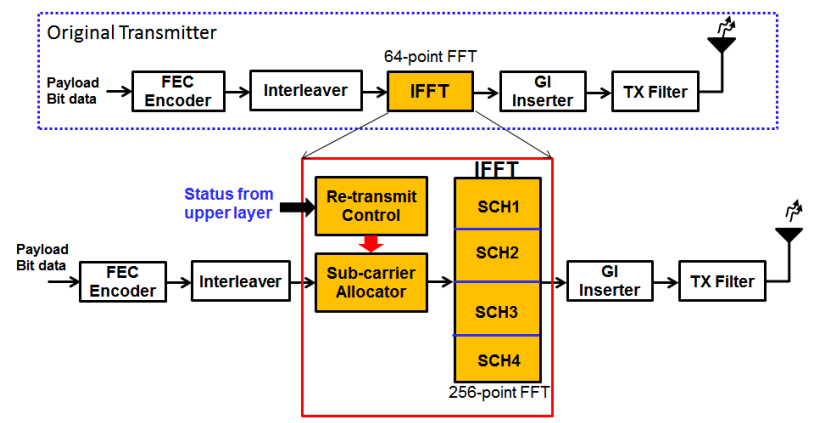

Fig. 5 Transceiver architecture

Meanwhile, the same concept is also applicable for modifying receiver system. The modification will be carried out in the subcarrier de-allocator module and corresponding controller after FFT processing. In addition, the same FFT block can be used for two modes, which are inverse FFT for transmission and normal mode for reception.

From the perspective of implementation complexity (with respect to hardware resource usage), the proposed scheme gives a lower area overhead and acceptable extra hardware cost. The circuit area overhead also corresponds to the amount of power consumption. This advantage is very important for system implementation in the field of industrial control system, where the system complexity (e.g. small form factor) and low power circuits are main considerations (in particular for STAs which are installed on the clients, e.g. industrial wireless robots).

\section{Performance Analysis}

In this section, we analyze the performance of our proposed retransmission scheme with channel selectivity. We first evaluate the performance on link level (e.g packet error rate) and then apply the results to obtain system level performance, in terms of system reliability, latency and control duration time.

\subsection{Link level performance}

Firstly, we define the analysis in single link transmission. In this case, we assume that the probability errors for DL and UL transmission are denoted by $P_{e}^{D L}$ and $P_{e}^{U L}$, respectively. The probability of a user will be fail in the initial transmission is considered as total probability error for round trip communication as given by Eq. (8):

$$
P_{e}=P_{e}^{D L}+P_{e}^{U L}-P_{e}^{D L} \times P_{e}^{U L}
$$


It should be noticed that in our case, the channel response in selected subchannel is almost similar (time invariant) within the control duration, unless we change the transmitted channel. Furthermore, we also assume that the probability error in each subchannel is uncorrelated, but have the same error distribution. Specifically, for the DL transmission, since we do not employ any channel selectivity, the PER on the $\tau$-th retransmission $P_{e}^{D L}(\tau)$ will remain the same as the initial transmission, $P_{e}^{D L}(1)$. On the other hand the probability for UL after $\tau$-th retransmission can be represented as:

$$
P_{e}^{U L}(\tau)=\left(P_{e}^{U L}\right)^{\tau}
$$

\subsection{System level performance: FAWLAN system case}

To assess the system level performance in wireless control systems, we evaluate the achievable control duration, which is considered a mandatory requirement in real-time factory automation system. This metric is calculated as average packet transmission time from a controller to endpoint clients (e.g. robots, motor, actuators, etc) at certain degree of reliability (e.g. PER). In order to evaluate the feasibility of the proposed retransmission scheme, we apply the proposed method to FAWLAN system, where one AP serves as controller for $N$ users. By definition, the AP communicates to all users periodically within control duration time, $T_{C D}$. This control duration includes synchronization time, $T_{\text {sync }}$, DL transmission time $T_{D L}$, UL transmission time $T_{U L}$, and several required retransmission time $T_{\text {recom }}$.

To achieve the target of system reliability performance, we expect that during working duration time $T_{w d}$, the probability of system error is less than a certain threshold value, $P_{e}^{t h}$. It is also assumed that during this working duration, the AP performs $\tau$ times of $T_{C D}$. In [9], it has been shown that to satisfy this requirement we should find an optimum value of retransmission number that satisfy Eq. (10):

$$
\begin{aligned}
& P_{e}(\Gamma) \times \frac{T_{w d}}{T_{C D}} \leq\left(P_{e}^{t h}\right) \\
& T_{C D}=T_{\text {sync }}+T_{S I F S}+T_{\text {com }}+\Gamma \times T_{\text {recom }}
\end{aligned}
$$

where $T_{S I F S}$ denotes Short Inter Frame Sequence that separates two consecutive frames, which is typically $16 \mu \mathrm{s}$. On the other hand, $T_{\text {com }}$ represents the total communication duration performed by the AP to all groups of STAs. $T_{\text {com }}$ takes into account $T_{D L}, T_{U L}$, and $T_{S I F S}$.

From Eq. (10) and (11), it is clear that to guarantee that the system becomes safe, there is a trade-off between probability error and control duration. To achieve lower probability error, we may apply large retransmission time. However, this will result in a longer control duration. The control duration per user is calculated from total control duration $T_{C D}$ after $\Gamma$ times of retransmission or target control duration elapsed over all associated users.

\section{Performance Results and Discussion}

\subsection{Simulation scenario}

The simulation study performed in this paper involves DL and UL transmission with retransmission diversity. The DL transmission employs Mixed VHT frame format and $80 \mathrm{MHz}$ bandwidth, while the UL transmission employs legacy format and $20 \mathrm{MHz}$ bandwidth. In addition, both DL and UL transmission also employ QPSK rate 1/2 coding scheme, one spatial stream, 0.25 Guard Interval ratio, and $3.2 \mu$ s OFDM symbol length. The DL transmission carries 48 byte data payload for each users and the UL transmission carries 32 bytes data payload, corresponding to $68 \mu$ s frame length (e.g. in the case of using $40 \mu \mathrm{s}$ PHY header).

The simulation environment assumed in this section incorporates the effects of hardware impairments, such as the nonlinearity of power amplifier (PA), carrier frequency offset for the RF part and quantization of the $\mathrm{AD} / \mathrm{DA}$ converter to take into account the real-world effect and to obtain a realistic performance. Additionally, in order to represent the industrial wireless network channel, we consider the indoor type channel model (e.g. Channel D). The distance between an AP and STAs (TX-RX distance) are set to $20 \mathrm{~m}$ to reflect the typical radius of factory control system. The summary of simulation environments are provided in Table 1 .

Table 1 Simulation environment

\begin{tabular}{|l|l|}
\hline Parameters & Values \\
\hline SNR & $10-20 \mathrm{~dB}$ \\
\hline TX - RX Distance & $20 \mathrm{~m}$ \\
\hline RF Carrier Frequency & $5.25 \mathrm{GHz}$ \\
\hline Phase Offset & 0.0842 degree \\
\hline Frequency Offset & $-13.675 \mathrm{ppm}$ \\
\hline ADC/DAC Resolution & $12 \mathrm{bits}$ \\
\hline Transmit Power & $17 \mathrm{~dB}$ \\
\hline Channel Model & Channel D (Indoor) \\
\hline
\end{tabular}

\subsection{UL PER performance results}

In this simulation, we assume that several number of channels (up to 4) could be used for channel selectivity. For example, '2 channel' refers to the transmitter of STA being allowed to use two different subchannels for retransmission. On the other hand, 'no selectivity' refers to the transmitter using a fixed subchannel.

First, we present the performance of UL transmission with several channel selectivity, as shown in Fig. 6 . It could be noticed that if there is no channel selectivity in short transmission duration (e.g. channel condition is stable), the performance of UL is not improved. Furthermore, when channel selectivity is employed, the PER performance is improved as the available channel for selectivity increases. As an example, at SNR $17 \mathrm{~dB}$, the PER 


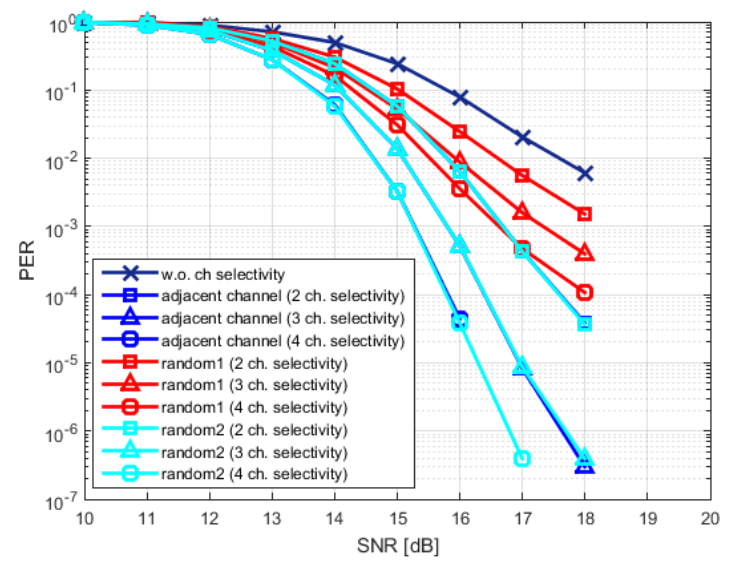

Fig. 6 PER performance of UL in different selectivity schemes

is improved from $2 \times 10^{-2}$ to $5 \times 10^{-6}$ by employing adjacent channel and permute channel (random2) selectivity when the number of selectivity of 4 channel. On the other hand, when employing random channel selectivity, the improvement of PER is only up to $5 \times 10^{-3}$. Hence, to achieve a target PER of $10^{-2}$, the required SNR can be reduced by around $2 \mathrm{~dB}$ when channel selectivity is employed.

The simulation results as shown in Fig. 6 also show that (1) the adjacent subchannel and random 2 selection method offer better reliability performance compared with random 1 subchannel selection, and (2) the performance of adjacent and random 2 is almost similar. These observations are reasonable since essentially these two methods utilize all available different subchannels for the next retransmission and do not use the previously employed one. On the other hand, the worse performance of random 1 subchannel selection is caused by reusing the same subchannel that previously has an error. In other words, this scheme limits the frequency diversity.

\subsection{Reliability-latency performance}

Furthermore, we also evaluate the trade-off between latency and reliability performance under various selectivity numbers in order to show the effectiveness of the proposed scheme in the context of URLLC system. For this evaluation, we use the success rate and normalized transmission time as performance metrics. Success rate represents the accumulated total correctly received packet, while the normalized transmission time is defined as the required time slot to transmit all packet data until correctly received at the receiver side. In this simulation, we use the obtained packet error rate of adjacent subchannel selection as previously presented in Fig. 6.

The reliability-latency performances for different target of success rate are shown in Fig. 7 and Fig. 8. As shown by Fig. 7, in order to achieve target success rate of 0.999 , the proposed retransmission using channel selectivity can reduce the transmission time (e.g. latency) from 10 transmission slots to up to 3 transmission slots (e.g. 2

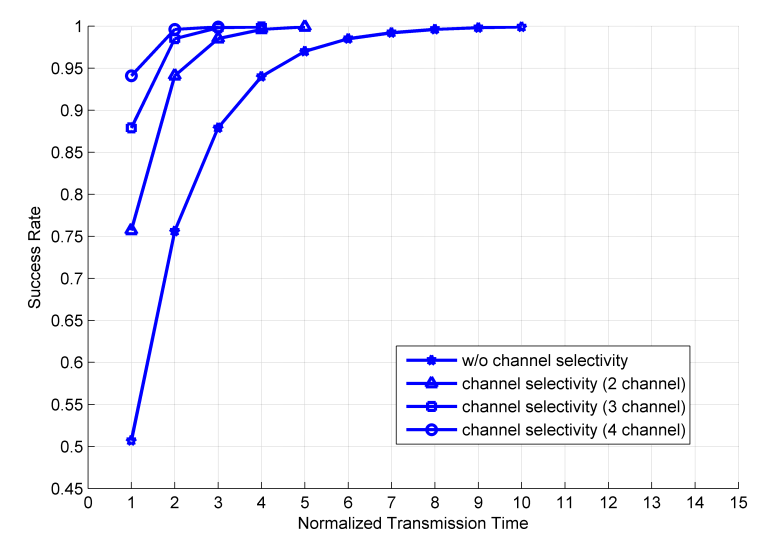

Fig. 7 Transmission reliability and latency performance with target success rate of 0.999

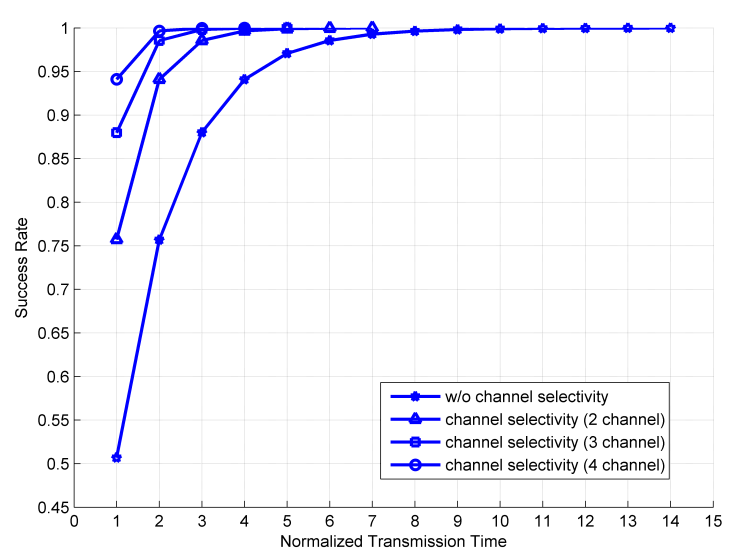

Fig. 8 Transmission reliability and latency performance with target success rate of 0.9999

transmission slot latency), when using 4 channel selectivity. On the other hand, to achieve target success rate of 0.9999 (shown by Fig. 8), the proposed scheme can reduce the transmission time from 14 transmission time slots to up to 4 transmission time slots. These results are equivalent to a reduction of transmission time of up to $70 \%$ as compared with the conventional one [9], where the selectivity schemes are not employed.

\subsection{Control duration performance on FAWLAN sys- tem}

In order to confirm applicability to such a realistic system, we employ the proposed retransmission scheme into the FAWLAN system. In this system level evaluation, we perform simulation by transmitting packet data to a large number of STAs (users), where the number users vary from 8 users up to 32 users. We also consider the error threshold is $P_{e}^{t h}=10^{-3}$ to evaluate the system error rate during long operations time $T_{w d}=1$ year to evaluate the requirement of safety level.

Fig. 9 shows achievable control duration for different SNR from the conventional FAWLAN system and the proposed retransmission scheme. This result implies that the proposed scheme significantly improve the control dura- 
tion performance, particularly for worse channel condition (lower SNR). On the other hand, in good channel condition (e.g. SNR higher than $18 \mathrm{~dB}$ ) both schemes offer almost similar control duration performance.

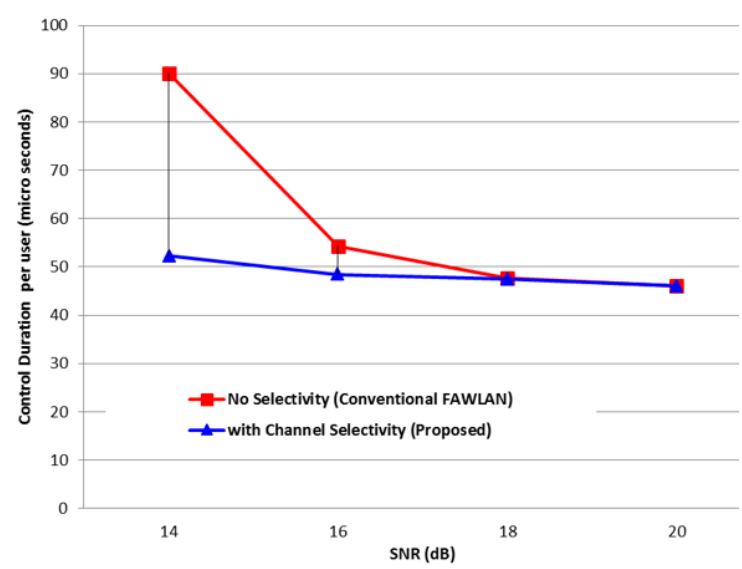

Fig. 9 Control duration per user in different SNR conditions

Finally, we evaluate the impact of channel selectivity on achievable control duration per user on different associated STAs (users). In this evaluation, we consider the lower SNR (e.g. $14 \mathrm{~dB}$ ) where the performance of channel selectivity is more beneficial. The results in Fig. 10 confirm that the retransmission with channel selectivity can reduce control duration by up to $36 \%$ (in average) from the conventional one for all cases of number of STAs. In addition, the control duration per user can achieve better results than the target of $100 \mu \mathrm{s}$. In the figure, the proposed method achieves a control duration per user from $52 \mu$ s to $63 \mu \mathrm{s}$.

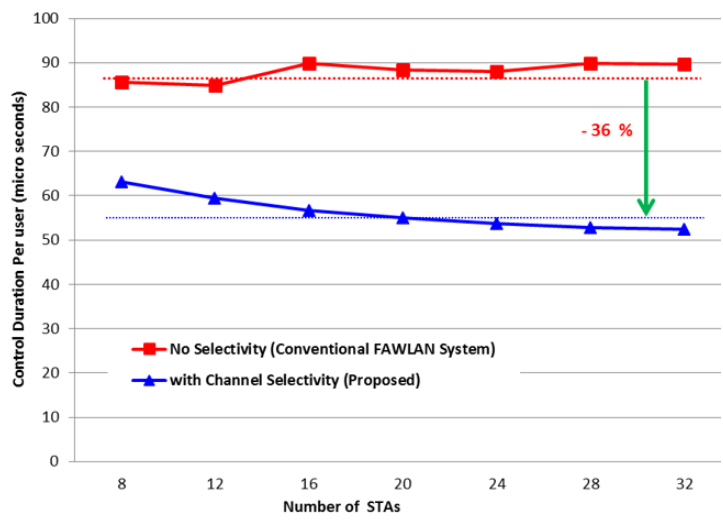

Fig. 10 Achievable control duration per user

\section{Conclusion}

In this paper, we have proposed a fast and low complexity retransmission scheme with channel selectivity for industrial wireless systems. The proposed retransmission scheme leverages the opportunity of frequency diversity by employing simple rescheduling of subcarrier allocation in OFDM transmission. The adjacent channel retransmission method offers better performance as compared with random1 method and has similar performance with random 2 method. The link level evaluation results show that the proposed scheme can achieve high success rate transmission and also at the same time can maintain the transmission latency. Furthermore, applying the proposed retransmission scheme in FAWLAN system could offer control duration per user less than $100 \mu$ s and reduce the control duration per user for approximately $36 \%$ from the conventional system. Therefore, the proposed retransmission scheme is potentially applicable in the transceiver of WLAN-based industrial wireless system, where the reliability and lowlatency communication are mandatory requirements.

\section{References}

[1] A. Frotzscher et al.: Requirements and current solutions of wireless communication in industrial automation, International Conference on Communication (ICC), pp. 67-72, June 2014.

[2] A. Willig: Recent and emerging topics in wireless industrial communications: A Selection, IEEE Transactions on Industrial Informatics, Vol. 4, Issue 2, pp. 102-124, May 2008.

[3] L. L. Bello and O. Mirabella: Communication techniques and architectures for Bluetooth networks in industrial scenarios, IEEE Conference on Emerging Technologies \& Factory Automation (ETFA), pp. 52-61, September 2005.

[4] N. Baker: ZigBee and Bluetooth strengths and weaknesses for industrial applications, Computing and Control Engineering Journal, Vol. 16, No. 2, pp. 20-25, April 2005.

[5] J. S. Lee, Y. W. Su and C. C. Shen: A comparative study of wireless protocols: Bluetooth, UWB, ZigBee, and Wi-Fi, Annual Conference of the IEEE on Industrial Electronics Society (IECON), pp. 46-51, November 2007.

[6] S. Petersen and S. Carlsen: Performance evaluation of wirelessHART for factory automation, IEEE Conference on Emerging Technologies \& Factory Automation (ETFA), pp. 1-9, September 2009.

[7] M. Weinar et al.: Design of a low-latency, high-reliability wireless communication system for control applications, IEEE International Conference on Communication (ICC), pp. 3835-3841, June 2014.

[8] M. Rentschler and P. Laukemann: Performance analysis of parallel redundant WLAN, IEEE Conference on Emerging Technologies \& Factory Automation (ETFA), pp. 1-8, September 2012.

[9] D. K. Lam et al.: A fast and safe industrial WLAN communication protocol for factory automation control systems, Transactions of the Institute of Systems, Control and Information Engineers, Vol. 29, Issue 1, pp. 29-39, January 2016.

[10] H. Rahul et al.: Frequency-aware rate adaptation and MAC protocols, Proceedings of the 15th Annual International Conference on Mobile Computing and Networking (MobiCom E9), pp. 193-204, September 2009.

[11] Y. Du et al.: SAMU: Design and implementation of selectivityaware MU-MIMO for wideband WiFi, 12th Annual IEEE International Conference on Sensing, Communication, and Networking (SECON), June 2015.

[12] X. Liu and H. Zhu: Novel packet retransmission in OFDMA systems using frequency diversity, IEEE Vehicular Technology Conference (VTC Spring), pp. 1-5, May 2011.

[13] V. Erceg et al.: TGn channel models, doc.: IEEE 802.11-03/940r4, May 2004 
[14] T. Paul and T. Ogunfunmi: Wireless LAN comes of age: Understanding the IEEE 802.11n Amendment, IEEE Circuit and System Magazine, pp. 28-54, First Quarter 2008.

[15] B. Holfeld et al.: Radio channel characterization at $5.85 \mathrm{GHz}$ for wireless M2M communication of industrial robots, IEEE Wireless Communications and Networking Conference, April 2016.

[16] R. Abreu et al.: A blind retransmission scheme for ultra-reliable and low latency communications, IEEE Vehicular Technology Conference (VTC Spring), pp. 1-5, June 2018.

[17] T. Watteyne et al.: Mitigating multipath fading through channel hopping in wireless sensor networks, IEEE International Conference on Communication (ICC), pp. 1-5, May 2010.

[18] A. M. Kurniawati et al.: Design of WLAN based system for fast protocol factory automation system, 22nd Asia-Pacific Conference on Communication (APCC), pp. 430-435, August 2016.

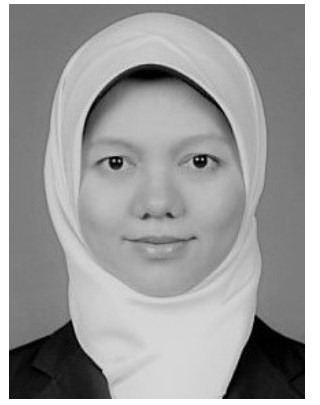

Astri Maria Kurniawati received her B.S. degree in electrical engineering and M.S. degree in biomedical engineering, from Bandung Institute of Technology, Indonesia in 2007 and 2012, respectively She is currently pursuing for her Ph.D. degree in Kyushu Institute of Technology, Japan. Her research interests are VLSI design and wireless system design.
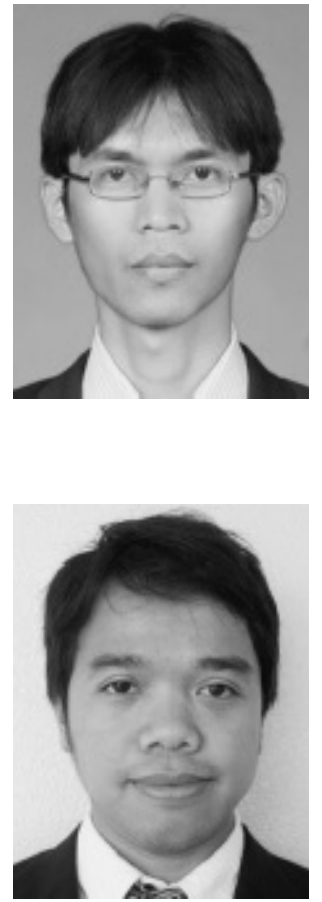

Leonardo Lanante Jr. received his B.S. degree in electronics and communications engineering and M.S. degree in electrical engineering both from University of the Philippines in 2005 and 2007, respectively. He received his Ph.D. degree in information systems from Kyushu Institute of Technology. $\mathrm{He}$ is currently an assistant professor in Kyushu Institute of Technology. His research interests include synchronization algorithms in wireless systems as well as signal processing in MIMO OFDM.

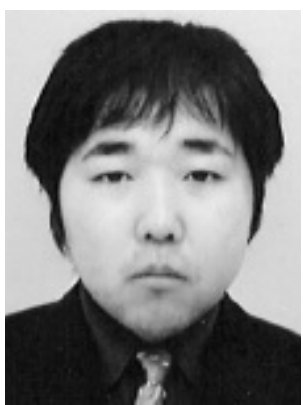

Yuhei Nagao received his M.E. and Ph.D. degrees from Kyushu Institute of Technology in 2006 and 2009, respectively. Since 2009, he has been with Kyushu Institute of Technology as a researcher. His research interests include wireless communication. $\mathrm{He}$ is a member of the IEEE.

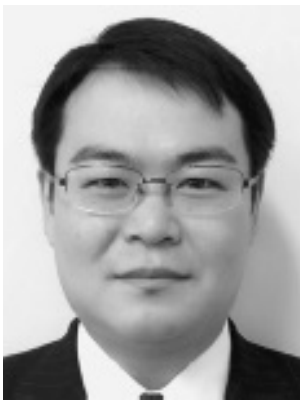

Masayuki Kurosaki received his B.E., M.E. and Ph.D. degrees from Tokyo Metropolitan University in 2000, 2002 and 2005, respectively. $\mathrm{He}$ was with Kyushu Institute of Technology from 2005 to 2011 as an assistant professor. Since 2011, he has been with Kyushu Institute of Technology as an Associate Professor. His research interests include image processing and wireless communication for multimedia. He is a member of the IEEE.

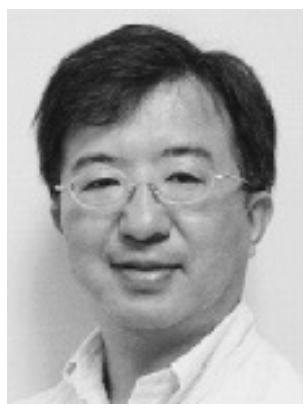

Hiroshi Ochi received his B.E. and M.E. degrees in electronics engineering from Nagaoka Institute of Technology, Japan in 1981 and 1984, respectively. He also received his Ph.D. degree in electrical engineering from Tokyo Metropolitan University in 1991. He was with University of the Ryukyus from 1986 until 1999 as an assistant and an associate professor. He also received MBA degree from Kyushu University in 2007. He is currently with Kyushu Institute of Technology as a Professor in computer and electronics engineering department from 1999. His current research interests include signal processing for wireless communication system, VLSI chip design and MOT education. He also organizes a venture company Radrix Co. Ltd. as a CEO.

(Received September 27, 2019; revised February 7, 2020) 